\title{
Nonlinear absorption and nonlinear refraction: maximizing the merit factors
}

\author{
M. Samoc ${ }^{\mathrm{a}, \mathrm{b} *}$, K. Matczyszyn ${ }^{\mathrm{a}}$, M. Nyk ${ }^{\mathrm{a}}$, J. Olesiak-Banska ${ }^{\mathrm{a}}$, D. Wawrzynczyk ${ }^{\mathrm{a}}$, P. Hanczyc ${ }^{\mathrm{a}, \mathrm{c}}, \mathrm{J}$. \\ Szeremeta $^{a}$, M. Wielgus ${ }^{\mathrm{a}}$, M. Gordel ${ }^{\mathrm{a}}$, L. Mazur ${ }^{\mathrm{a}, \mathrm{e}}$, R. Kolkowski ${ }^{\mathrm{a}, \mathrm{f}}$ B. Straszak ${ }^{\mathrm{a}}$, M.P. Cifuentes ${ }^{\mathrm{d}}$, \\ M.G. Humphrey ${ }^{\mathrm{d}}$ \\ anstitute of Physical and Theoretical Chemistry, Wroclaw University of Technology, Wroclaw, \\ Poland \\ ${ }^{\mathrm{b}}$ Laser Physics Centre, The Australian National University, Canberra, Australia \\ ${ }^{c}$ Department of Physical Chemistry, Chalmers University of Technology, Gothenburg, Sweden \\ ${ }^{d}$ Research School of Chemistry, The Australian National University, Canberra, Australia \\ ${ }^{\mathrm{e}}$ Laboratoire de Chimie des Polymeres, Université Pierre et Marie Curie- Paris VI, France \\ ${ }^{\mathrm{f}}$ Laboratoire de photonique quantique et moléculaire, École Normale Supérieure de Cachan, France
}

\begin{abstract}
Both nonlinear absorption and nonlinear refraction are effects that are potentially useful for a plethora of applications in photonics, nanophotonics and biophotonics. Despite substantial attention given to these phenomena by researchers studying the merits of disparate systems such as organic materials, hybrid materials, metal-containing molecules and nanostructures, it is virtually impossible to compare the results obtained on different materials when varying parameters of the light beams and different techniques are employed. We have attempted to address the problem by studying the properties of various systems in a systematic way, within a wide range of wavelengths, and including the regions of onephoton, two-photon and three-photon absorption.

The objects of our studies have been typical nonlinear chromophores, such as $\pi$-conjugated molecules, oligomers and polymers, organometallics and coordination complexes containing transition metals, organometallic dendrimers, small metal-containing clusters, and nanoparticles of various kinds, including semiconductor quantum dots, plasmonic particles and rare-earth doped nanocrystals. We discuss herein procedures to quantify the nonlinear response of all of these systems, by defining and comparing the merit factors relevant for various applications.
\end{abstract}

Keywords: Nonlinear absorbers, nonlinear refractive index, nonlinear microscopy, 3D information storage, all-optical switching

\section{INTRODUCTION}

Despite considerable effort given to investigations of the cubic nonlinear optical properties of various materials, only a few practical materials are finding applications in nonlinear refraction-based devices. The number of substances and nanosystems considered for and actually finding use as nonlinear absorbers is considerably larger. However, whether for nonlinear refraction or for nonlinear absorption, which are both related to the complex third-order susceptibility $\chi^{(3)}(-\omega ; \omega,-\omega, \omega)$, there are few general guidelines and little related experimental data that could be used for optimization of the materials performance $\left(\mathrm{cf}^{1}\right)$. The only exception is in the case of nonlinear absorbers used as two-photon excited fluorescence emitters, because for such fluorophores there exists a large body of experimental data on the spectral dependence of their nonlinear absorption cross-sections obtained through measurements of action spectra of the twophoton excited fluorescence ${ }^{2,3}$. However, this technique is unsuitable for investigations of species that show no or little luminescence, e.g. those to be used for multiphoton-induced photochemistry, optical power limiting, etc. For many of these materials, nonlinear absorption and/or nonlinear refraction data are only available at discrete wavelengths, which may be in various relations to the known energy levels of the substance.

*marek.samoc@pwr.wroc.pl; phone +48713204466; fax +48713203364; www.organometallics.pwr.wroc.pl 
In addition, the large body of data that has been published in the literature is of little use for systematic comparisons between the various nonlinear materials. Firstly, many investigations carried out with long (eg nanosecond) laser pulses, or with shorter laser pulses but at exceedingly high repetition rates, have given results that contain difficult-to-quantify contributions from various cumulative effects such as thermal effects due to local heating of the sample, excited-state absorption, contributions from photochemically-produced species (eg radicals, charge carriers, photoisomerization products and intermediates). Secondly, the data are often presented in a way that makes it difficult to assess the relative merits of different species, eg they refer to macroscopic nonlinearities of mixtures (solutions) containing different concentrations of the nonlinear chromophores.

We discuss herein the requirements for proper assessment of the NLO properties of materials considered for various applications of third-order NLO effects, in particular those involving nano-objects such as nanoparticles.

\section{EXPERIMENTAL}

\subsection{Measurement technique}

Although there are several ways to determine the real and imaginary part of the $\chi^{(3)}(-\omega ; \omega,-\omega, \omega)$ susceptibility, most results in the literature on the development of new cubic NLO materials are those from Z-scan measurements ${ }^{4}$. The technique is well known and very easy to implement, and the analysis of data is straightforward. However, since the results are obtained without time resolution, considerable care has to be taken to avoid confusing the contributions of processes occurring on different time scales.

In the implementations of Z-scan which have provided the results discussed here, we used three different experimental set-ups: the first at the Laser Physics Centre, Research School of Physics and Engineering, at the Australian National University in Canberra, Australia, the second at the Institute of Physical and Theoretical Chemistry, Wroclaw University of Technology, Wroclaw, Poland and the third at the Research School of Chemistry, at the Australian National University. All three experimental facilities used laser systems consisting of a Ti-sapphire regenerative amplifier and an optical parametric amplifier to provide high energy ( hundreds of $\mu \mathrm{J})$, low repetition rate $(100-1000 \mathrm{~Hz})$, tunable $(\sim 500-2000 \mathrm{~nm})$ femtosecond range (100 - $200 \mathrm{fs})$ pulses of light. The first system used a Clark-MXR CPA2010 RGA and a Light Conversion TOPAS OPA while the other two systems used Quantronix Integra RGAs and Quantronix Palitra OPAs. The typical Z-scan experiments required some spatial and spectral filtering of the OPA output and attenuation of the pulses to $\sim 1 \mu \mathrm{J}$ before they were focused with the Z-scan lens to a spot usually in the range $\mathrm{w}_{0}=20-60 \mu \mathrm{m}$, the spot size being adjusted to ensure that the Rayleigh range of the beam was greater than the total thickness of the sample under investigation, in order for the thin sample approximation to be fulfilled.

The data obtained from the Z-scan experiments were analyzed as described in ref ${ }^{5}$ to obtain both the macroscopic and microscopic NLO parameters characterizing the investigated materials.

\subsection{Nonlinear data}

As mentioned above, nonlinear refraction and nonlinear absorption are both described by the complex susceptibility, its real part being responsible for the refraction and its imaginary part for the absorption. Despite some work that has been carried out to understand the relations between the spectral dependencies of the two components ${ }^{6-8}$, it is not currently possible to predict the nonlinear refraction solely from nonlinear absorption measurements. For measurements of nonlinear chromophores in liquid solutions and solid matrices (for example, a polymer matrix), it should be emphasized that nonlinear absorption is usually determined without the background of contributions from the host, while nonlinear refraction always consists of contributions of both the host and the guest and also, in the case of measurements of the investigated material in a container (eg a glass cuvette), from the container walls. In effect, the nonlinear refraction parameters are often determined with lower accuracy, especially for measurements covering both the resonant and nonresonant regions in which the off-resonant refractive nonlinearity may be difficult to estimate.

The primary results of Z-scan measurements are usually expressed as the macroscopic parameters characterizing the investigated samples: the nonlinear refractive index $\mathrm{n}_{2}$ and the nonlinear absorption coefficient $\alpha_{2}$. If pure onecomponent materials are investigated then these are the parameters relevant for practical applications and it is straightforward to compare the merits of various materials. However, these parameters are of little use if, as is often the case, solutions or guest-host systems are investigated. Two ways of presenting the data then appear sensible: 
- presentation of the nonlinear optical parameters for (a possibly hypothetical) pure material composed of only the active component. Such extrapolated $\mathrm{n}_{2}$ and $\alpha_{2}$ may be obtained by simple extrapolation to $100 \%$ of the active component, although the procedure is not strictly accurate because of the assumption of linear dependence of the properties of the material on its composition expressed in, for example, weight fractions or volume fractions or mole fractions may be far from reality. However, even if such an extrapolation is not rigorous, it does provide an estimate of the merit of the active component.

- The data can be presented in the form of microscopic properties of molecules or particles forming the material. The usual way of presenting the cubic nonlinear optical properties is by quoting the real and imaginary parts of the cubic hyperpolarizability $\gamma(-\omega ; \omega,-\omega, \omega)$ and, in the case of nonlinear absorption, the two-photon absorption cross section $\sigma_{2}$ (usually in Goeppert-Mayer units: $1 \mathrm{GM}=10^{-50} \mathrm{~cm}^{4} \mathrm{~s}$ ).

Comparisons between different nonlinear species that can be used to create new multicomponent materials or that can be used as probes in some investigated systems (eg biological ones) are not straightforward, since they may depend both on the actual application intended for the material and on additional factors such as the practical maximum content of the active NLO chromophore in a material and the robustness of the system (eg susceptibility to bleaching).

\section{EXAMPLES OF RESULTS}

We compare here experimental results obtained by us on several classes of materials, all of them containing atoms of metals bound in various ways. Although the chemistry of organometallic compounds (molecules in which a carbon-metal bond exists), coordination complexes (where a metal ion is surrounded by ligands that typically use oxygen or nitrogen to bind to the metal), polynuclear clusters, lanthanide-containing nanoparticles, semiconductor quantum dots and plasmonic metal nanoparticles may seem very different, from the practical point of view their merits need to be compared in order to decide on the best system for a given application.

The data available from multi-wavelength $\mathrm{Z}$-scan can be presented as spectra of macroscopic or microscopic parameters. Figure 1 shows results obtained for an organometallic dendrimer ${ }^{9} 1,3,5-\left(\right.$ trans $-\left[\mathrm{Ru}\left(\mathrm{C} \equiv \mathrm{CC}_{6} \mathrm{H}_{4}-4-\mathrm{NO}_{2}\right)(\mathrm{dppe})_{2}\left(\mathrm{C} \equiv \mathrm{CC}_{6} \mathrm{H}_{4}\right.\right.$ $4-\mathrm{C} \equiv \mathrm{C})])_{3} \mathrm{C}_{6} \mathrm{H}_{3}$. This is a highly nonlinear compound in which the molecular hyperpolarizability reaches values of the order of $10^{-31} \mathrm{esu}$. The data in Figure 1 were obtained by simple extrapolation of the results obtained at the concentration of 0.1 weight $\%$ to the concentration of $100 \%$ using the formulas $\mathrm{n}_{2 \text {,solution }}=\mathrm{gn}_{2 \text {,solute }}+(1-\mathrm{g}) \mathrm{n}_{2 \text {,solvent }}$ and $\alpha_{2 \text {,solution }}=$ $\mathrm{g \alpha}_{2, \text { solute, }}$, where $\mathrm{g}$ is the weight fraction of the chromophore in solution. It should be kept in mind that relationships of this kind that are often used in the literature, whether using weight fraction as above, volume fraction or molar fraction, are only order-of-magnitude estimates for properties of hypothetical materials. A better approximation may be obtained by using a type of "oriented gas" approach in which the properties of materials are calculated from additivity of molecular properties, accounting for changes of local fields. As discussed in Ref. ${ }^{10}$, such an approach leads to the conclusion that the relationship between the value of the linearly extrapolated $\mathrm{n}_{2}$ and the real value for a pure substance should be:

$$
\frac{n_{2, \text { extrap. }}}{n_{2}}=\left(\frac{L_{\text {solv. }}}{L_{\text {subst. }}}\right)^{4}\left(\frac{n_{\text {subst. }}}{n_{\text {solv. }}}\right)^{2} \frac{\rho_{\text {solv. }}}{\rho_{\text {subst. }}} C_{\text {or }}
$$

where $L=\frac{n^{2}+2}{3}$ is the Lorentz local field factor, $n$ is the refractive index, $\rho$ is the density and $C_{o r}$ is the correction for the possible difference in the orientational order of molecules in solution and in the solid. Table 1 shows three examples of expected ratios of the extrapolated and true values of $\mathrm{n}_{2}$. In the first and third example the solvent parameters are those of $\mathrm{CHCl}_{3}$ and in the second case those of THF. The solute parameters for the first two examples are those expected for an organometallic molecule while the third case is that expected for a semiconductor quantum dot. Obviously, the biggest influence on the relevance of such extrapolated results is that of the value of the refractive index of the materials. These, however, are difficult to predict, since the refractive index of a material consisting of, for example, colloidal quantum dots may be very different from that of bulk semiconductor. 
Table 1. Examples of ratios of extrapolated and real values of the nonlinear refractive index

\begin{tabular}{|lllll|}
\hline $\mathrm{n}_{\text {solv. }}$ & $\mathrm{n}_{\text {subst }}$ & $\rho_{\text {solv }}$ & $\rho_{\text {subst }}$ & $\mathrm{n}_{\text {2extr }} / \mathrm{n}_{2}$ \\
\hline 1.446 & 1.7 & 1.48 & 1 & 1.00 \\
1.404 & 1.7 & 0.89 & 1 & 0.567 \\
1.446 & 3 & 1.48 & 4 & 0.0304 \\
\hline
\end{tabular}

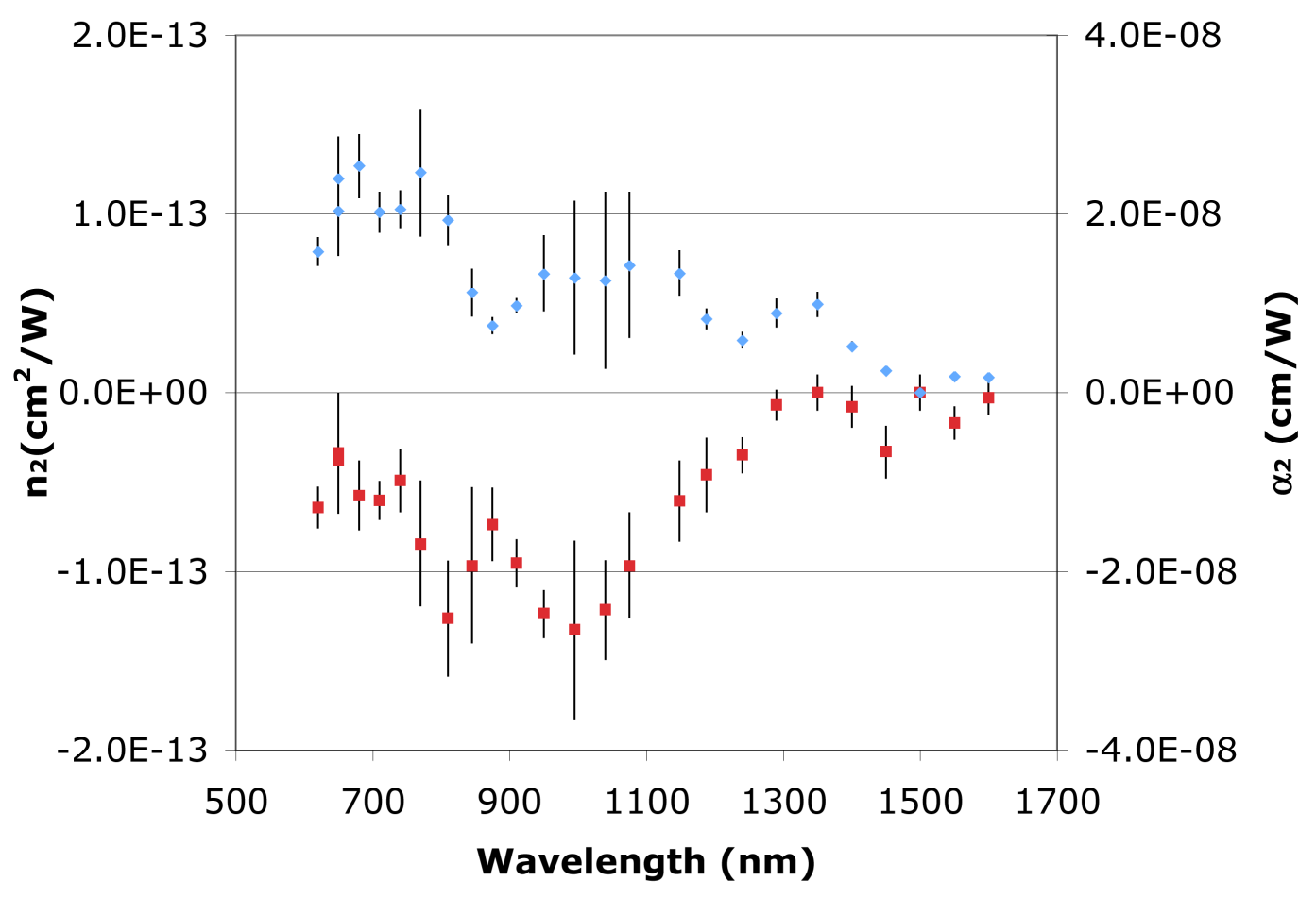

Figure 1. Nonlinear refractive index and nonlinear absorption coefficient dispersion for $1,3,5-\left(\right.$ trans- $\left[\mathrm{Ru}\left(\mathrm{C} \equiv \mathrm{CC}_{6} \mathrm{H}_{4}-4-\right.\right.$ $\left.\left.\left.\mathrm{NO}_{2}\right)(\text { dppe })_{2}\left(\mathrm{C} \equiv \mathrm{CC}_{6} \mathrm{H}_{4}-4-\mathrm{C} \equiv \mathrm{C}\right)\right]\right)_{3} \mathrm{C}_{6} \mathrm{H}_{3}$ (converted data presented in Ref. ${ }^{9}$ )

It should be remarked that similar behavior is expected for the nonlinear absorption coefficient, which may formally be considered to be related to the imaginary part of the nonlinear refractive index taken as the complex quantity, i.e. $\alpha_{2}=\frac{4 \pi}{\lambda} \kappa^{(2)}=\frac{4 \pi}{\lambda} \operatorname{Im}\left(\hat{n}_{2}\right)$.

\section{MERIT FACTORS}

\subsection{Nonlinear absorption merit factors}

To consider optimization of materials for application of their nonlinear absorption properties one needs first to specify in what form the materials will be actually used. Three possibilities exist here:

- the nonlinear species is used in the form of neat bulk material

- the nonlinear species is used in liquid or solid solution at an optimized concentration 
- the nonlinear species is used as a single entity, eg as a single molecule or nanoparticle

The second consideration is that of the physical process that is to be optimized. Three major applications of nonlinear absorption are the most common:

- use of nonlinear absorbers for light-intensity dependent attenuation of a laser beam, eg in power limiting applications

- use of nonlinear absorbers for initiation of a photochemical reaction, eg in two-photon induced photopolymerization

- use of nonlinear absorbers for generation of luminescence through a nonlinear absorption process followed by (upconverted) emission of light.

Table 2 lists merit factors that should be used to assess the suitability of various nonlinear absorbing species for such applications, depending on their physical form. In the case of bulk nonlinear absorbers, the relevant quantity is basically the macroscopic nonlinear absorption coefficient, although more advanced comparisons of various materials should also take into account the influence of the linear (and possibly nonlinear) refractive index on factors such as reflection losses, distortion of the focused beam, the possibility of focusing to a small spot, etc. The damage threshold or the threshold of other, unwanted nonlinear processes may also be important. For photochemistry and for the use of a material as a twophoton excited luminophore, the nonlinear absorption should be multiplied by the quantum efficiency of the relevant process: $\eta_{\text {phot }}$ represents the efficiency of photogeneration of the desired photochemical species while $\eta_{\text {lum }}$ is the quantum yield of luminescence photons. At the microscopic scale, the quantity to consider is the two-photon absorption crosssection, but what really matters is the product of the cross-section and the concentration of nonlinearly absorbing species per unit volume $N$. Since $N=1 / V=N_{A} / V_{m}$, where $N_{A}$ is the Avogadro number, $V$ is the molecular volume, and $V_{m}$ is the molar volume of the species, we conclude that $\sigma_{2} / \mathrm{V}$ is the relevant merit factor. It should be noted that this merit factor was postulated recently ${ }^{11}$ as a more appropriate one than the often-used two-photon absorption cross-section scaled by the molecular weight $\sigma_{2} / \mathrm{M}$. The relation between these two merit factors is as follows: $\sigma_{2} / \mathrm{V}=\left(\sigma_{2} / \mathrm{M}\right)\left(\rho / \mathrm{N}_{\mathrm{A}}\right)$.

Table 2. Figures of merit for nonlinear absorbers

\begin{tabular}{|c|c|c|c|}
\hline $\begin{array}{l}\text { Process/Form of } \\
\text { nonlinear absorber }\end{array}$ & Neat substance & Solution & Nanoobject \\
\hline Nonlinear absorption & $\alpha_{2} \propto \mathrm{N \sigma}_{2} \propto \sigma_{2} / \mathrm{V}$ & $\alpha_{2} \propto N_{\max } \sigma_{2}$ & $\sigma_{2}$ \\
\hline $\begin{array}{l}\text { Two-photon absorption } \\
\text { induced } \\
\text { photochemistry }\end{array}$ & $\begin{array}{l}\alpha_{2} \eta_{\text {phot }} \propto N \sigma_{2} \eta_{\text {phot }} \\
\propto \sigma_{2} \eta_{\text {phot }} / V\end{array}$ & $\alpha_{2} \eta_{\text {phot }} \propto N_{\max } \sigma_{2} \eta_{\text {phot }}$ & $\sigma_{2} \eta_{\text {phot }}$ \\
\hline $\begin{array}{l}\text { Two-photon induced } \\
\text { luminescence }\end{array}$ & $\begin{array}{l}\alpha_{2} \eta_{\text {lum }} \propto N \sigma_{2} \eta_{\text {lum }} \\
\propto \sigma_{2} \eta_{\text {lum }} / \mathrm{V}\end{array}$ & $\alpha_{2} \eta_{\text {lum }} \propto N_{\max } \sigma_{2} \eta_{\text {lum }}$ & $\sigma_{2} \eta_{\text {lum }}$ \\
\hline
\end{tabular}

The situation is different in the case of nonlinear species in liquid or solid solutions at moderate or low concentrations. The optimization of such absorbers should be in seeking the optimal product of the molecular two-photon absorption cross-section and the maximum concentration at which it can be used. Such a limitation of concentration can simply come from limited solubility of the species in the required matrix, but, in the case of nonlinear absorption excited luminophores, the limitation may also come from concentration quenching of the emission.

Finally, the situation is different again, if the nonlinear absorber is to be used in the form of a nano-object, eg for imaging of single nanoparticles bound to biological molecules. The quantity of importance is then simply the crosssection for the whole nano-object (eg a dendrimer containing many chromophores bound to it, the whole quantum dot or a Au nanorod). In the imaging applications, there is, however, another important factor that must be taken into account, which is the robustness of the nonlinear absorber, i.e. its resistance to bleaching. Basically, the quality of an image which may be obtained depends on the number of photons that can be emitted by a species upon nonlinear excitation, before it is bleached eg by a photochemical reaction. Such quantitative data are not widely available yet for a variety of nonlinear 
absorbers used for imaging in nonlinear microscopy, but should be collected for meaningful comparisons between various disparate species used or proposed for these applications.

Representative data on the $\sigma_{2} / \mathrm{M}$ and $\sigma_{2} / \mathrm{V}$ merit factors for several classes of nonlinear absorbers are presented in Table $3^{12}$.

Table 3. Examples of merit factors for several classes of nonlinear absorbers.

\begin{tabular}{|c|c|c|c|c|}
\hline Species & $\begin{array}{l}\text { Wavelength } \\
(\mathrm{nm})\end{array}$ & $\sigma_{2}(\mathrm{GM})$ & $\begin{array}{l}\sigma_{2} / \mathrm{M} \\
(\mathrm{GM} \\
\mathrm{mol} / \mathrm{g})\end{array}$ & $\begin{array}{l}\sigma_{2} / \mathrm{V} \\
\left(\mathrm{GM} / \AA^{3}\right)\end{array}$ \\
\hline $\begin{array}{l}\text { N-cored Ru-containing } \\
\text { dendrimer }\end{array}$ & 580 & $1.06 \times 10^{4}$ & 1.13 & 1.1 \\
\hline $4.2 \mathrm{~nm}$ CdSe quantum dots & 1000 & $6 \times 10^{4}$ & 0.13 & 0.47 \\
\hline $\begin{array}{l}\text { Upconverting } 30 \mathrm{~nm} \mathrm{NaYF} 4 \\
\text { nanoparticles doped with } \\
2 \% \mathrm{Er}^{3+}, 20 \% \mathrm{Yb}^{3+}\end{array}$ & 980 & $8 \times 10^{6}$ & 0.1 & 0.25 \\
\hline $10 \mathrm{~nm} \times 35 \mathrm{~nm}$ gold nanorods & $\sim 600$ & $5 \times 10^{8}$ & 15 & 170 \\
\hline
\end{tabular}

\subsection{Nonlinear refraction merit factors.}

A consideration of the merit factors for nonlinear refraction applications should commence from recalling the fact that in the most important application of this effect (all-optical switching), the main obstacle for its implementation remains the need to avoid both excessive linear absorption and excessive nonlinear absorption. The one-photon absorption merit factor for all-optical switching is $W=\frac{n_{2} I_{\max }}{\alpha \lambda}$ and the two-photon absorption merit factor can be written as $\frac{1}{T}=\frac{n_{2}}{\alpha_{2} \lambda}$ where $I_{\max }$ is the maximum light intensity that can be used for the process ${ }^{13}$. These factors should be maximized, with values greater than unity needed for the process to be feasible at all. As discussed in Ref. ${ }^{14}$ the one-photon merit factor is in fact related to two technological parameters of crucial relevance for the implementation of nonlinear optical switches: the length of the device and the light intensity needed for accomplishing the switching.

The merit factors that should be used for comparison between various materials, and at various wavelengths of operation, are listed in Table 4. The two merit factors listed above can be recast in terms of the microscopic properties available from measurements on solutions, i.e. the real and imaginary parts of the hyperpolarizability $\gamma$. However, since the macroscopic nonlinearity involves the influence of the refractive index, its role should also be included. The merit factor $\mathrm{W}$ can be seen to depend on the real part of the hyperpolarizability scaled by the linear absorption, represented here by the molar absorption coefficient $\varepsilon_{\mathrm{m}}$. It is also important to include in the comparisons the influence of the linear refractive index, both in the local field factors and as the $1 / n^{2}$ factor needed to convert between the macroscopic susceptibility $\chi^{(3)}$ and the nonlinear refractive index $n_{2}$. The T merit factor can be represented by the ratio of the real and imaginary parts of the cubic hyperpolarizabilities, no local field corrections being required in this case. Finally, the merit of a nonlinear refractive material needs to be expressed as the value of available $n_{2}$ itself, which is the third entry in Table 4. 
Table 4. Suggested merit factors for comparisons of nonlinear refraction materials.

\begin{tabular}{|l|l|l|}
\hline Merit factor & Neat material & Solution \\
\hline $\begin{array}{l}\text { One photon absorption merit } \\
\text { factor }\end{array}$ & \multicolumn{2}{|c|}{$\mathrm{W} \propto \mathrm{n}_{2} / \alpha \propto \mathrm{L}^{4} \operatorname{Re}(\gamma) / \mathrm{n}^{2} \varepsilon_{\mathrm{m}}$} \\
\hline $\begin{array}{l}\text { Two-photon absorption merit } \\
\text { factor }\end{array}$ & \multicolumn{1}{|c|}{$1 / \mathrm{T} \propto \mathrm{n}_{2} / \alpha_{2} \propto \operatorname{Re}(\gamma) / \operatorname{Im}(\gamma)$} \\
\hline Maximum available nonlinearity & $\mathrm{L}^{4} \mathrm{NRe}(\gamma) / \mathrm{n}^{2} \propto \mathrm{N} \mathrm{L}{ }^{4} \operatorname{Re}(\gamma) / \mathrm{n}^{2} \mathrm{~V}$ & $\mathrm{~L}^{4} \mathrm{~N}_{\max } \operatorname{Re}(\gamma) / \mathrm{n}^{2}$ \\
\hline
\end{tabular}

\section{ACKNOWLEDGMENTS}

This work was supported by the Foundation for Polish Science under the "Welcome" programme, by the Polish Ministry of Science and Higher Education under the Grant no. N N507 599038, by the Polish-French "cotutelle" PhD programme and by the Australian Research Council (ARF to M.P.C. and APF to M.G.H.).

\section{REFERENCES}

[1] Christodoulides, D. N., Khoo, I. C., Salamo, G. J., Stegeman, G. I. and Van Stryland, E. W., "Nonlinear refraction and absorption: mechanisms and magnitudes", Adv. Opt. Photon. 2, 60-200 (2010).

[2] Xu, C. and Webb, W. W., "Measurement of two-photon excitation cross sections of molecular fluorophores with data from 690 to $1050 \mathrm{~nm} "$, J. Opt. Soc. Am. B, 13, 481-491 (1996).

[3] Makarov, N. S., Drobizhev, M. and Rebane, A., "Two-photon absorption standards in the 550-1600 nm excitation wavelength range", Opt. Express 16, 4029-4047 (2008).

[4] Sheikh-bahae, M., Said, A. A., Wei, T., Hagan, D. J. and Van Stryland, E. W., "Sensitive Measurement of Optical Nonlinearites Using a Single Beam", IEEE J. Quantum Electr. 26, 760-769 (1990).

[5] Samoc, M., Samoc, A., Dalton, G. T., Cifuentes, M. P., Humphrey, M. G. and Fleitz, P. A., "Two-photon absorption spectra and dispersion of the complex cubic hyperpolarizability $\gamma$ in organic and organometallic chromophores", Multiphoton Processes in Organics and Their Application, edited by I. Rau and F. Kajzar, 341-355, Old City Publishing, Philadelphia, 2011.

[6] Sheik-Bahae, M., "Nonlinear Optics Basics. Kramers-Kronig Relations in Nonlinear Optics", Encyclopedia of Modern Optics, edited by R. D. Guenther, Academic Press, Amsterdam, 2005.

[7] Samoc, M., Samoc, A., Humphrey, M. G., Cifuentes, M. P., Luther-Davies, B. and Fleitz, P. A., "Z-Scan Studies of Dispersion of the Complex Third-Order Nonlinearity of Nonlinear Absorbing Chromophores", Mol. Cryst. Liq. Cryst. 485, 894-902 (2008).

[8] Samoc, M., Samoc, A., Dalton, G. T., Cifuentes, M. P., Humphrey, M. G. and Fleitz, P. A., "Dispersion of the complex cubic nonlinearity in two-photon absorbing organic and organometallic chromophores", Proc. SPIE-Int. Soc. Opt. Eng. 6801, 68011O-68011-68016 (2008).

[9] Samoc, M., Corkery, T. C., McDonagh, A. M., Cifuentes, M. P. and Humphrey, M. G., "Organometallic Complexes for Non-linear Optics. 49. Third-Order Non-linear Optical Spectral Dependence Studies of Arylalkynylruthenium Dendrimers", Aust. J. Chem. 64, 1267-1271 (2011).

[10] Samoc, M., Samoc, A., Luther-Davies, B., Bao, Z., Yu, L., Hsieh, B. and Scherf, U., "Femtosecond Z-scan and degenerate four-wave mixing measurements of real and imaginary parts of the third-order nonlinearity of soluble conjugated polymers", J. Opt. Soc. Am. B, 15, 817-825 (1998).

[11] Schwich, T., Cifuentes, M. P., Gugger, P. A., Samoc, M. and Humphrey, M. G., "Electronic, Molecular Weight, Molecular Volume, and Financial Cost-Scaling and Comparison of Two-Photon Absorption Efficiency in Disparate 
Molecules (Organometallic Complexes for Nonlinear Optics. 48.) - A Response to "Comment on 'Organometallic Complexes for Nonlinear Optics. 45. Dispersion of the Third-Order Nonlinear Optical Properties of TriphenylamineCored Alkynylruthenium Dendrimers.' Increasing the Nonlinear Response by Two Orders of Magnitude."", Adv. Mat. 23, 1433-1435 (2011).

[12] Samoc, M., Matczyszyn, K., Nyk, M., Olesiak-Banska, J., Wawrzynczyk, D., Hanczyc, P., Szeremeta, J., Wielgus, M., Humphrey, M. G. and Cifuentes, M. P., "Nonlinear absorption and refraction in metal-containing chromophores and nano-objects", Chem. Phys. Letters, submitted (2011).

[13] Stegeman, G. I. and Torruellas, W. E., "Nonlinear materials for information processing and communications.", Philos. Trans. R. Soc. Lond. Phys. Sci. Eng., 745-756 (1996).

[14] Samoc, M., "Third-order nonlinear optical materials: practical issues and theoretical challenges", J. Mol. Model. 17, 2183-2189 (2011). 